\title{
Polymorphisms in thioredoxin genes are associated with prenatal thyroid hormone status in a cohort of high fish-eating pregnant women
}

\author{
A. J. Yeates ${ }^{1}$, T. Love $^{2}$, K. Engstrom ${ }^{3}$, M. S. Mulhern ${ }^{1}$, E. M. McSorley ${ }^{1}$, G. Watson ${ }^{2}$, \\ G. E. Myers' ${ }^{2}$, E. van Wijngaarden ${ }^{2}$, P. W. Davidson ${ }^{2}$, C. Shamlaye ${ }^{4}$, J. J. Strain ${ }^{1}$ \\ and K. Broberg ${ }^{3}$ \\ ${ }^{1}$ Northern Ireland Centre for Food and Health, University of Ulster, Coleraine, BT52 1SA, ${ }^{2}$ University of Rochester, \\ School of Medicine and Dentistry, NY, USA, ${ }^{3}$ Lund University, Sweden and ${ }^{4}$ Ministry of Health, Victoria, Mahé, \\ Republic of Seychelles
}

Iodine and selenium are required for synthesis of the thyroid hormones, thyroxine (T4) and triiodothyronine (T3), which are critical for optimal neurodevelopment ${ }^{(1)}$. Maternal T4 has previously been shown to positively correlate with child cognitive development in the Seychelles Child Development Study Nutrition Cohort 1 (SCDS NC1) ${ }^{(2)}$. The selenoproteins thioredoxin $(T X N)$ and thioredoxin reductases (TXNRD 1 and 2) protect the thyroid from oxidative stress during hormone synthesis ${ }^{(3)}$. The aim of the present study was to investigate associations between single nucleotide polymorphisms (SNPs) in thioredoxin genes, $T X N$ and $T X N R D$, with prenatal status of thyroid hormones in the SCDS NC1.

Blood was collected from pregnant women at enrolment and at 28 weeks gestation, from which serum concentrations of thyroid hormones were previously measured and genotyping performed for selected SNPs following leukocyte DNA extraction $(n=170)$. Univariate analysis of covariance (ANCOVA) was used to compare associations of each genotype with serum hormone concentrations, whilst adjusting for maternal age, BMI and smoking status. For $T X N$, homozygote minor and heterozygote alleles were combined.

\begin{tabular}{|c|c|c|c|c|c|c|c|c|c|}
\hline \multirow[b]{3}{*}{ Gene and Alleles } & \multirow[b]{3}{*}{$n$} & \multicolumn{8}{|c|}{ Thyroid hormones (pmol/L) } \\
\hline & & \multicolumn{2}{|c|}{ T4 enrolment } & \multicolumn{2}{|c|}{ T4 28 wks } & \multicolumn{2}{|c|}{ T3 enrolment } & \multicolumn{2}{|c|}{ T3 28 wks } \\
\hline & & Mean & SE & Mean & SE & Mean & SE & Mean & $\mathrm{SE}$ \\
\hline \multicolumn{10}{|l|}{ TXNRD1 (rs11111979) } \\
\hline Homozygous minor $G / G$ & 20 & $10 \cdot 93$ & 0.54 & $8 \cdot 63$ & $0 \cdot 27$ & $4 \cdot 81$ & $0 \cdot 25$ & 3.79 & $0 \cdot 12$ \\
\hline $\begin{array}{l}\text { Heterozygous G/C } \\
\text { TXNRD2 (rs5748469) }\end{array}$ & 58 & $12 \cdot 07^{*}$ & 0.32 & $9 \cdot 04^{*}$ & $0 \cdot 16$ & $5 \cdot 22$ & $0 \cdot 15$ & 3.66 & 0.07 \\
\hline Homozygous minor AlA & 19 & $11 \cdot 31$ & 0.59 & $9 \cdot 19$ & $0 \cdot 29$ & 4.93 & $0 \cdot 27$ & 3.93* & $0 \cdot 13$ \\
\hline $\begin{array}{l}\text { Heterozygous ClA } \\
T X N(\mathbf{r s 1 0 4 9 9 2 7 )}\end{array}$ & 51 & 11.89 & $0 \cdot 36$ & $8 \cdot 59$ & $0 \cdot 18$ & $5 \cdot 17$ & $0 \cdot 16$ & 3.74 & 0.08 \\
\hline Heterozygous $G / A$ & 12 & $13 \cdot 24 * *$ & 0.69 & $9 \cdot 64^{*}$ & 0.35 & $6 \cdot 07 * *$ & $0 \cdot 31$ & $4 \cdot 05^{*}$ & $0 \cdot 14$ \\
\hline
\end{tabular}

Values are estimated means and their standard errors (SE). Significant differences in means between each allele compared to the reference genotypes for TXNRD1, 2 and TXN (CC, CC and AA respectively) are shown $(* P<0 \cdot 05, * * P<0 \cdot 01)$ from ANCOVA adjusting for maternal age, BMI and smoking.

Minor allele frequency of TXNRD1, TXNRD2 and TXN were calculated as $37 \%, 39 \%$ and $4 \%$ respectively. Variations of each genotype were significantly associated with greater concentrations of thyroid hormones. TXNRD1 heterozygous was associated with T4 at both time-points, with $T X N R D 2$ homozygous being associated with T3 at 28 wks and $T X N$ heterozygous with T4 and T3 at both time-points. Results indicate that genetic variation in $T X N$ genes may influence prenatal thyroid hormone concentrations in this cohort of high-fish eating pregnant women. Further research should confirm these results in a larger cohort and investigate the influence of genetic variation in selenoproteins on neurodevelopment.

This work was supported by grants from the US National Institute of Environmental Health Sciences, the National Institutes of Health and the Government of Seychelles.

1. Schomburg L \& Kohrle J (2008) Mol Nutr Food Res 52, 1235-1246.

2. Davidson PW, Strain JJ, Myers GJ et al. (2008) Neurotoxicol 29, 767-775.

3. Leoni SG, Kimura ET, Santisteban P et al. (2011) Molec Endocrinol 25, 1924-1935. 